\title{
A spin-filter device based on armchair graphene nanoribbons
}

\author{
A. Saffarzadeh 1,2 , 困 and R. Farghadan ${ }^{3}$ \\ ${ }^{1}$ Department of Physics, Payame Noor University, Nejatollahi Street, 159995-7613 Tehran, Iran \\ ${ }^{2}$ Computational Physical Sciences Laboratory, Department of Nano-Science, \\ Institute for Research in Fundamental Sciences (IPM), P.O. Box 19395-5531 Tehran, Iran \\ ${ }^{3}$ Department of Physics, Tarbiat Modares University, P.O. Box 14115-175 Tehran, Iran
}

(Dated: November 11, 2018)

\begin{abstract}
The coherent spin-polarized electron transport through a zigzag-edge graphene flake (ZGF), sandwiched between two semi-infinite armchair graphene nanoribbons, is investigated by means of Landauer-Buttiker formalism. To study the edge magnetism of the ZGF, we use the half-filled Hubbard model within the Hartree-Fock approximation. The results show that the junction acts as a spin filter with high degree of spin polarization in the absence of magnetic electrodes and external fields. By applying a gate voltage the spin-filtering efficiency of this device can be effectively controlled and the spin polarization can reach values as high as $90 \%$.
\end{abstract}

Graphene nanoribbons (GNRs) and graphene junctions are good candidates for electronic and spintronic devices due to high carrier mobility, long spin-relaxation times and lengths, and spin-filtering effect [1-3]. In fact, the conduction electrons in carbon-based materials can move very long distances without scattering due to their small spin-orbit coupling and low hyperfine interaction. For instance, in GNRs with zigzag edges, electronic transport is dominated by edge states which have been observed in scanning tunneling microscopy [4]. These states are expected to be spin-polarized and make zigzag-edge GNR (ZGNR) junctions attractive for nanoscale spintronic applications such as spin filters [5-12]. In order to achieve a spintronic device, it is very important to find nonmagnetic materials where a spin-polarized current can be injected and flowed without becoming depolarized. The ground state of ZGNRs has an antiferromagnetic spin configuration where the total spin $(S)$ is zero. However, when the system has different number of A- and B-sublattice sites, the total spin of the ground state is $2 S=N_{A}-N_{B}$ [13] and by appropriate designing, one can form a ferromagnetic spin configuration at the zigzag edges. Most of the previous studies on spinfiltering effects have been focused on the junctions, which consist of ZGNR electrodes.

In this letter, a GNR junction which operates as a spin-filter in the absence of an external electric field is presented and the influence of edge atoms on spin transport through the junction will be examined. We also investigate the sensitivity of the spin polarization to the gate voltage to obtain a maximum value for this polarization. An interesting feature of our system is that, in spite of the previous studies, the type of electrodes in this junction is armchair-edge GNR and the central part of the system is a zigzag-edge graphene nanodisk [6, 14], as shown in Fig. 1(a). In this junction, the right GNR electrode with a ribbon index $n=8$ has a width $W_{R}=0.86$ $\mathrm{nm}$, while the left electrode with a ribbon index $n=33$

*Electronic address: a-saffar@tpnu.ac.ir
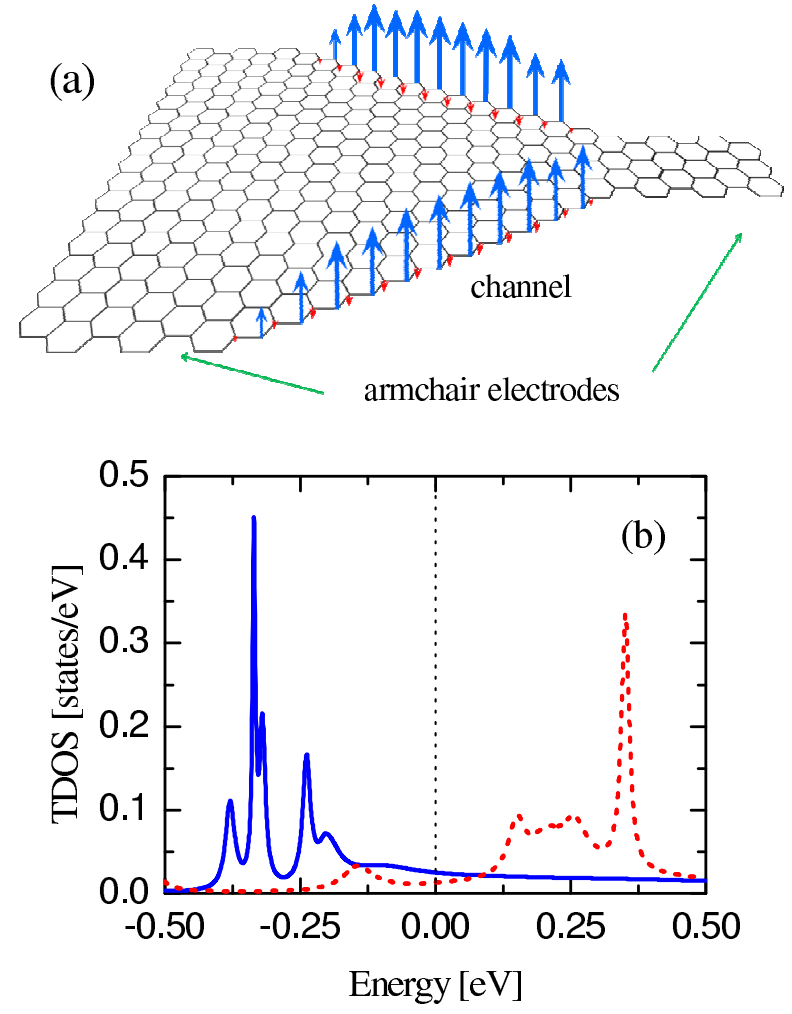

FIG. 1: (Color online) (a) The spin-filter device. The device shows that the localized magnetic moments only form on the edge atoms of the channel and the electrodes are nonmagnetic. The upward (blue) arrows correspond to the majority spins, while the downward (red) arrows correspond to the minority spins. (b) The majority (solid line) and minority (dashed line) spin densities at the channel. The vertical dotted line indicates the Fermi energy.

has a width $W_{L}=3.93 \mathrm{~nm}$. Furthermore, the central region (nanodisk) with a trapezoidal shape consists of $N_{C}=240$ carbon atoms and produces a ferromagnetic spin configuration at its edges.

We simulate the system depicted in Fig. 1(a) by use of a single-band tight-binding model and an on-site Hub- 
bard repulsion treated in the mean-field approximation at half-filling to account for electron-electron interaction in the junction and calculate the localized moments on the zigzag-edge atoms. Thus, the mean field Hamiltonian can be written as [15]

$\hat{H}_{C}=\sum_{i, j, \sigma}\left(\epsilon_{i} \delta_{i, j}-t\right) \hat{d}_{i \sigma}^{\dagger} \hat{d}_{j \sigma}+U \sum_{i, \sigma}\left\langle\hat{n}_{i,-\sigma}\right\rangle\left[\hat{n}_{i, \sigma}-\frac{1}{2}\left\langle\hat{n}_{i, \sigma}\right\rangle\right]$,

where $\hat{d}_{i \sigma}^{\dagger}$ and $\hat{d}_{i \sigma}$ are the electron creation and annihilation operators, respectively, and $\hat{n}_{i, \sigma}=\hat{d}_{i \sigma}^{\dagger} \hat{d}_{i \sigma}$ is the number operator for an electron with spin $\sigma$ at site $i$. Here, $\epsilon_{i}$ will be set to zero except in the gated region (channel) where it is equal to gate potential $V_{G} . t=2.66$ $\mathrm{eV}$ is the transfer integral between all the nearest neighbor sites and $U=1.06 t$ is the on-site Coulomb interaction. Starting from an antiferromagnetic configuration as an initial condition and considering the effect of the semiinfinite GNRs [16] on the connected atoms of the channel, we solve the mean-field Hamiltonian self-consistently by iteration method. Therefore, the Green's function and the spin density on each atom of the channel should be calculated iteratively until a convergence of the spin density is reached. The Green's function of the nanodisk is expressed as

$$
\hat{G}_{C}(\omega)=\left[(\omega+i \eta) \hat{I}-\hat{H}_{C}-\hat{\Sigma}_{L}-\hat{\Sigma}_{R}\right]^{-1},
$$

where $\eta$ is a positive infinitesimal and $\Sigma_{L, R}$ are the selfenergy matrices due to the connection of left and right GNR electrodes to the channel. The spin-dependent density of states and the expectation value for the number operator of electron on each site of the channel are given as

$$
\begin{gathered}
g_{i \sigma}(\omega)=-\frac{1}{\pi} \operatorname{Im}\left\langle i \sigma\left|\hat{G}_{C}(\omega)\right| i \sigma\right\rangle, \\
\left\langle\hat{n}_{i \sigma}\right\rangle=\int_{-\infty}^{E_{F}} g_{i \sigma}(\omega) d \omega .
\end{gathered}
$$

Accordingly, the spin at each site of the channel can be expressed as $S_{i}=\frac{\left\langle\hat{n}_{i \uparrow}\right\rangle-\left\langle\hat{n}_{i \downarrow}\right\rangle}{2}$. In the mean-field level, there is not a spin-flip scattering or any other interactions. Therefore, the spin-polarized transport through such a junction can be considered within the coherent regime [17]. In the coherent transport, the spindependent conductance at low temperature can be written as $G_{\sigma}=\frac{e^{2}}{h} \operatorname{Tr}\left[\hat{\Gamma}_{L} \hat{G}_{C} \hat{\Gamma}_{R} \hat{G}_{C}^{\dagger}\right]_{\sigma}$, where $\hat{\Gamma}_{\alpha}$, the coupling matrices, can be expressed as $\hat{\Gamma}_{\alpha}(\omega)=-2 \operatorname{Im}\left[\hat{\Sigma}_{\alpha}(\omega)\right]$.

The total density of states (TDOS) per site for each spin subband, $\frac{1}{N_{C}} \sum_{i=1}^{N_{C}} g_{i, \sigma}(\omega)$, in the central region and in the presence of the electrodes has been shown in Fig. 1(b). The spin-dependent electronic states inside the energy window are completely separated into majority and minority spin subbands and in the other energy ranges (not shown) are approximately degenerate. The difference between the two spin subbands explains the localized magnetization in the channel edges. In such a case,

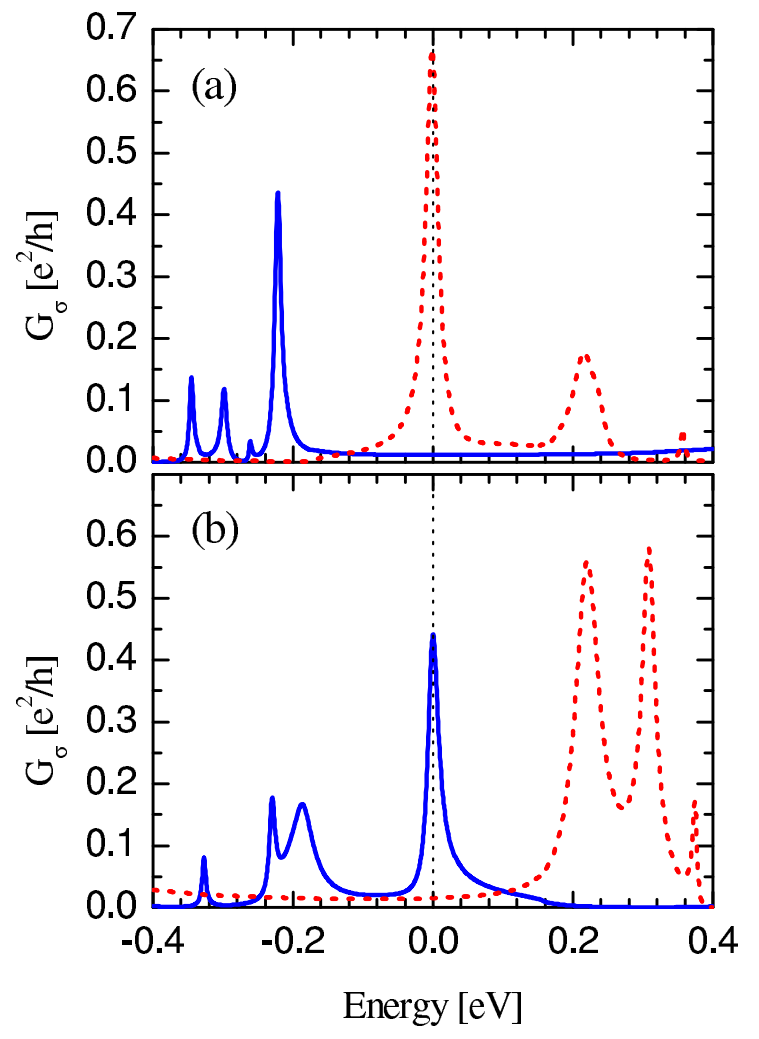

FIG. 2: (Color online) (a) Spin-dependent conductance as a function of energy at (a) $V_{G}=0.36 \mathrm{~V}$ and (b) $V_{G}=-1.82 \mathrm{~V}$. The solid (dashed) line is for majority (minority) spin electrons. The vertical dotted line indicates the Fermi energy.

the total spin of the channel reaches $S=2.82$, which has a maximum value $S=0.13$ in the middle of the channel edges [shown in Fig. 1(a)]. We see that the TDOS for spin-up electrons is more localized at lower energies than the spin-down ones. The separation between these two spin subbands which provide two paths for electron conduction through the junction well explains the spin-filter effect of the system. In fact, the importance of designing such a device is that the system at ground state is spin-polarized without applying external fields.

It has been well known that an armchair edge does not induce local edge magnetism [15]. Therefore, the electron conduction through the armchair-edge electrodes is not spin-polarized, but when the electron arrives at the nanodisk region, different scatterings occur for the electronic waves with different spin densities. Therefore, the electron conduction for both spin-up and spin-down electrons is different at certain energies and the generation and manipulation of the spin-polarized current would be possible. We should note that because of the special design for this junction there is not a significant difference between ferromagnetic and antiferromagnetic configurations, both for the electronic structure and the magnetic moment of the atoms on the edge of the ZGF, hence the transport properties for two spin configurations are the same. 


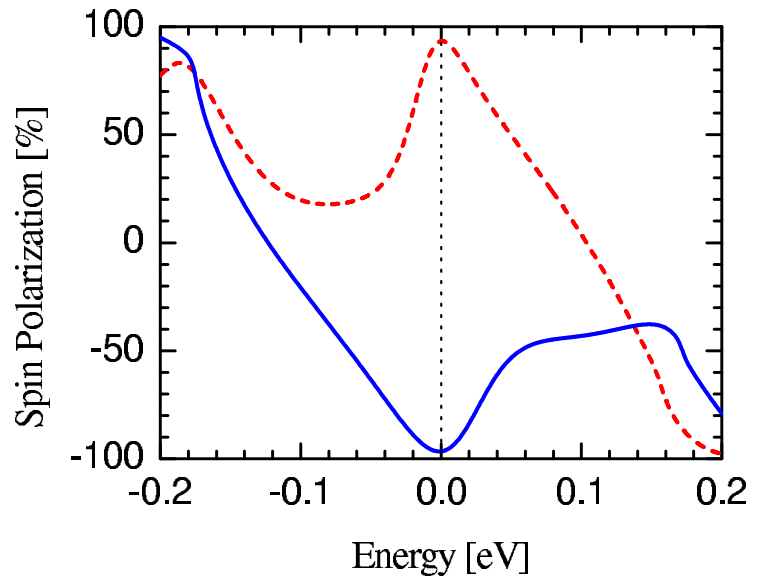

FIG. 3: (Color online) (a) Degree of spin polarization as a function of energy at two different gate voltages. The solid line is for $V_{G}=0.36 \mathrm{~V}$ and the dashed line is for $V_{G}=-1.82 \mathrm{~V}$.

The gate control of spin conduction is an important feature, which can clearly demonstrate high spin-filter effect in our device and perhaps in future all-carbon circuits [12]. Applying a gate voltage changes the potential energy in atomic sites and accordingly, the electronic states are shifted and slightly modified (due to the electrodes) by this effect. Hence, the transmission channels in the system may significantly vary [17, 18]. In a ZGNR, a gate potential may shift the spin-up and spin-down states differently and therefore, spin filters and spin switches can be achieved without magnetic contacts. [6, 19, 20]. For this purpose, we have shown in Fig. 2 the spin-dependent conductance as a function of energy for majority and minority electrons at two different gate voltages $V_{G}=0.36 \mathrm{~V}$ and $V_{G}=-1.82 \mathrm{~V}$. We have chosen these values because the spin-filtering effect in the system can be easily demonstrated. We can see that the conductance spectrum of two spin subbands is approximately separated in all electronic states within the energy window, same as the TDOS shown in Fig. 1(b). At the positive gate voltage $\left(V_{G}=0.36 \mathrm{~V}\right)$, the transmission channel of minority spin electrons is fully open at the Fermi energy, while that for majority spin electrons is effectively blocked. Such a feature will be reversed if the negative gate voltage $\left(V_{G}=-1.82 \mathrm{~V}\right)$ is applied.

To obtain the spin-filtering efficiency, we have shown in Fig. 3 the degree of spin polarization for electrons traversing the channel which can be defined as $P=$ $\frac{G_{\uparrow}(\omega)-G_{\downarrow}(\omega)}{G_{\uparrow}(\omega)+G_{\downarrow}(\omega)}$. In this figure, the dip at the Fermi energy clearly represents that the minority spin electrons at $V_{G}=0.36 \mathrm{~V}$ carry most of the current, while the peak is related to the majority spin electrons at $V_{G}=-1.82 \mathrm{~V}$. This can be easily understood from comparing the behavior of spin-dependent conductance of majority and minority spin electrons. As seen in Fig. 2, when majority (minority) spin electrons show high peaks in their conductance spectrum, the conduction of minority (majority) electrons approximately vanishes, i.e. high degree of spin polarization. The spin polarization just like the conductance spectra is sensitive to changes of energy and in some energy ranges there are high spin polarizations for each spin subband. We should note that, with considering the more number of carbon atoms in the channel the total magnetic moment increases and the spin-filter efficiency is enhanced. Also, the spin density of each site is calculated at zero temperature and with considering the effect of temperature in the number operator Eq.(41) [6] the magnetic moment at the ZGF reduces and this reduction is able to change the efficiency of this spin filter device. Perhaps the most interesting aspect of our system is the ability for generation of spin-polarized currents in the absence of traverse fields and using electrodes that are nonmagnetic.

In summary, based on the non-equilibrium Green function technique and the mean-field Hubbard model, we proposed a spin filter device which preferably passes only one type of spin currents through the system. In such a device, the current is spin-polarized due to the finite-size effect and geometry of ZGF. The spin-filtering efficiency could be controlled by a gate voltage so that the spin polarization can be higher than $90 \%$ which shows that the system acts as a spin switch.

The authors would like to thank J. J. Palacios for helpful discussions.
[1] S. Sanvito, Nature Nanotechnology 2, 204 (2007).

[2] W.Y. Kim, K.S. Kim, Nature Nanotechnology 3, 408 (2008).

[3] Y.-W. Son, M. L. Cohen, and S. G. Louie, Nature (London) 444, 347 (2006); 446, 342 (2007).

[4] Y. Kobayashi, K. Fukui, T. Enoki, K. Kusakabe, Phys. Rev. B 73, 125415 (2006); Y. Niimi, T. Matsui, H. Kambara, K. Tagami, M. Tsukada, H. Fukuyama, Phys. Rev. B 73, 085421 (2006).

[5] X. Jia, M. Hofmann, V. Meunier, B.G. Sumpter, J. Campos-Delgado, J.M. Romo-Herrera, H. Son, Y-P Hsieh, A. Reina, J. Kong, M. Terrones, M.S. Dressel- haus, Science 323, 1701 (2009).

[6] J. Guo, D. Gunlycke, and C. T. White, Appl. Phys. Lett. 92, 163109 (2008).

[7] J. Guo and Y. Ouyang, Appl. Phys. Lett. 94, 243104 (2009).

[8] H. Kumazaki and D. S. Hirashima, J. Phys. Soc. Jpn. 78 094701 (2009).

[9] Y. Hancock, A. Uppstu, K. Saloriutta, A. Harju, and M. J. Puska, Phys. Rev. B 81, 245402 (2010).

[10] X. Lü, Y. Zheng, H. Xin, and L. Jiang, Appl. Phys. Lett. 96, 132108 (2010).

[11] T. Ozaki, K. Nishio, H. Weng, and H. Kino, Phys. Rev. 
B 81, 075422 (2010).

[12] M. G. Zeng, L. Shen, Y. Q. Cai, Z. D. Sha, and Y. P. Feng, Appl. Phys. Lett. 96, 042104 (2010).

[13] E. H. Lieb, Phys. Rev. Lett. 62, 1201 (1989).

[14] J. Fernández-Rossier and J. J. Palacios, Phys. Rev. Lett. 99, 177204 (2007).

[15] M. Fujita, K. Wakabayashi, K. Nakada, and K. Kusakabe, J. Phys. Soc. Jpn. 65, 1920 (1996).

[16] M. P. López Sancho, J. M. López Sancho and J. Rubio,
J. Phys. F: Met. Phys. 14, 1205 (1984); 15, 851 (1985).

[17] S. Datta, Electronic Transport in Mesoscopic Systems (Cambridge University Press, Cambridge, UK, 1995).

[18] A. Saffarzadeh, J. Appl. Phys. 103, 083705 (2008).

[19] A. Saffarzadeh, J. Appl. Phys. 104, 123715 (2008).

[20] S. Lakshmi, S. Roche, and G. Cuniberti, Phys. Rev. B 80, 193404 (2009). 